\title{
Mixing in Heavy Quark Effective Field Theory
}

\section{Citation}

Georgi, Howard. 1992. “Mixing in Heavy Quark Effective Field Theory.” Physics Letters B 297 (34): 353-57. https://doi.org/10.1016/0370-2693(92)91274-d.

\section{Permanent link}

http://nrs.harvard.edu/urn-3:HUL.InstRepos:41467469

\section{Terms of Use}

This article was downloaded from Harvard University's DASH repository, and is made available under the terms and conditions applicable to Other Posted Material, as set forth at http:// nrs.harvard.edu/urn-3:HUL.InstRepos:dash.current.terms-of-use\#LAA

\section{Share Your Story}

The Harvard community has made this article openly available.

Please share how this access benefits you. Submit a story.

Accessibility 
\#HUTP-92/A049

$9 / 92$

\title{
$D-\bar{D}$ Mixing in Heavy Quark

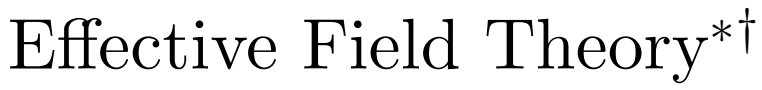

\author{
Howard Georgi \\ Lyman Laboratory of Physics \\ Harvard University \\ Cambridge, MA 02138
}

\begin{abstract}
I analyze $D-\bar{D}$ mixing using the techniques of heavy quark effect field theory. [1] The analysis suggests that the there may be important cancellations among the dispersive effects of different kinds of final states, so that the total mixing may be considerably smaller than previous estimates. [2, 3]
\end{abstract}

*Research supported in part by the National Science Foundation under Grant \#PHY-8714654.

${ }^{\dagger}$ Research supported in part by the Texas National Research Laboratory Commission, under Grant \#RGFY9206. 


\section{Introduction}

In the classic analyses of $D-\bar{D}$ mixing in the standard model, Donoghue et al. [2] and Wolfenstein [3] argued that the short distance contributions to the mixing are negligible compared to "dispersive" contributions from second order weak interactions with mesonic intermediate states,

$$
\sum_{I} \frac{\left\langle D^{0}\left|H_{W}\right| I\right\rangle\left\langle I\left|H_{W}\right| \overline{D^{0}}\right\rangle}{m_{D}^{2}-m_{I}^{2}+i \epsilon}
$$

In particular, because of the large $S U(3)$ breaking in $D^{0}$ decays, the contributions of particular intermediate states to (1.1) is apparently much larger than the contribution of the box diagram, shown in figure 1. This is quite different from the situation in $K-\bar{K}$ mixing, where the box diagram, shown in figure 2, is thought to be comparable to the dispersive contributions. This difference may be very important phenomenologically. If the dispersive contribution to $D-\bar{D}$ mixing is as large as suggested in [2, 3], the reach of $D-\bar{D}$ mixing as a signal for new physics beyond the standard model is reduced. In other words, when $D-\bar{D}$ mixing is finally observed experimentally, it will be important to have a good estimate of the standard model contribution, so that we will know whether we are seeing standard model physics or new physics beyond the standard model.

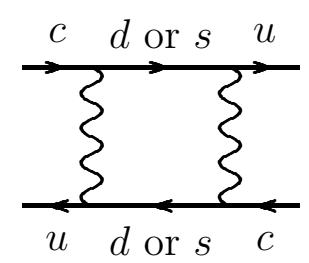

Figure 1: The box graph for $D-\bar{D}$ mixing.



Figure 2: The box graph for $K-\bar{K}$ mixing.

Here, we reexamine the issue of $D-\bar{D}$ mixing, making use of the heavy quark effective field theory (HQEFT). [1] We will find a puzzle. We will identify an enhancement of a well-defined 


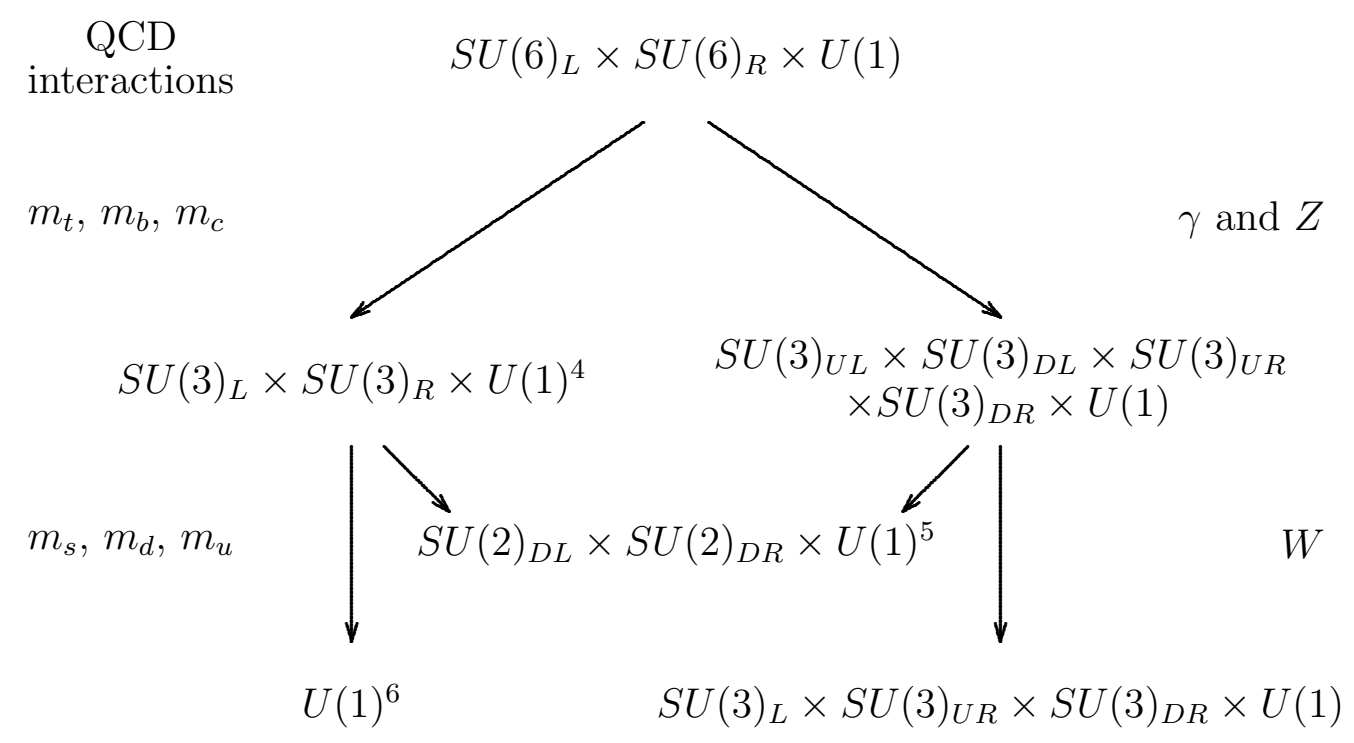

Figure 3: Quark flavor symmetry.

set of "long-distance" contributions. However, the enhancement does not appear to be as large as suggested by [2, 3]. If the heavy quark analysis is at all valid, it suggests that the total dispersive contribution is considerably smaller than the contributions found in [2, [3] from specific final states. This is certainly possible. Contributions from various different classes of intermediate states can cancel to give a smaller total mixing. It may be that the HQEFT analysis implies that such a cancellation will take place.

When we use the heavy quark effective theory to analyze $D$ meson properties, we are assuming that $m_{c}$ is much greater than $\Lambda_{Q C D}$. Thus it would not be completely surprising if the results of the heavy quark analysis were misleading, but at least it is a well defined approximation from which you can discuss corrections. At any rate, we will assume in the following that we can treat the $c$ quark as heavy.

\section{Symmetries of the Standard Model}

Before we discuss the HQEFT analysis in detail, I will discuss the flavor symmetry structure of $D-\bar{D}$ mixing. I want to make a point of this, because the enhancements that we find for the long-distance contributions will be intimately connected to their flavor symmetry properties. The symmetry structure of the standard model is shown diagrammatically in figure 3 . 
The $S U(6)_{L} \times S U(6)_{R} \times U(1)$ symmetry of the QCD interactions acting on six massless quarks is broken by the "large" quark masses, $m_{t}, m_{b}$, and $m_{c}$ down to $S U(3)_{L} \times S U(3)_{R} \times U(1)^{4}$ and by the interactions of the $\gamma$ and $Z$ down to $S U(3)_{U L} \times S U(3)_{D L} \times S U(3)_{U R} \times S U(3)_{D R} \times U(1)$. We will be particularly interested in the common subgroup which is left invariant by both, $S U(2)_{D L} \times$ $S U(2)_{D R} \times U(1)^{5}$, under which the left- and right-handed $d$ and $s$ transform as doublets. In particular, the transformation properties of the $\Delta c=1$, charm-changing nonleptonic weak interactions, $H_{W}$, under the left- and right-handed $U$-spin,

$$
S U(2)_{D L} \times S U(2)_{D R}
$$

will play a crucial role in our discussion. The point is that if we ignore the small mixing to the third family $\left(s_{2}, s_{3} \approx 0\right)$, we can write the $\Delta c=1$ Hamiltonian at the $W$ scale in the form

$$
\frac{G_{F}}{\sqrt{2}} \overline{\psi_{L}} \gamma^{\mu} u_{L} \vec{\kappa} \cdot \vec{\tau} \overline{c_{L}} \gamma_{\mu} \psi_{L}
$$

where $\psi_{L}$ is the two component $S U(2)_{D L}$ doublet

$$
\psi_{L}=\left(\begin{array}{l}
s_{L} \\
d_{L}
\end{array}\right)
$$

and $\vec{\kappa}$ is the complex constant $U$-spin vector

$$
\vec{\kappa}=\frac{1}{2}\left(\begin{array}{c}
c \\
i \\
s
\end{array}\right)
$$

with

$$
c=c_{1}^{2}-s_{1}^{2}, \quad s=2 s_{1} c_{1}
$$

While the detailed form of the weak Hamiltonian changes as you go down to lower scales, [4] the transformation properties under (2.2) do not, at least not above $1 \mathrm{GeV}$, because the QCD interactions do not break the symmetry. Thus the $\Delta c=1$ weak Hamiltonian is proportional to the $U$-spin vector, $\vec{\kappa}$, and the $\Delta c=2$ mixing is proportional to two factors of $\vec{\kappa}$. This is relevant because $\vec{\kappa}$ has the interesting property

$$
\vec{\kappa} \cdot \vec{\kappa}=0 .
$$

This is the underlying group theoretical statement of the GIM [5] suppression in $D-\bar{D}$ mixing. To make a nonvanishing $U$-spin singlet, we need a $U$-spin breaking "spurion" in the form of an 
additional $U$-spin vector $\vec{A}$ to form a non-zero combination, $(\vec{A} \cdot \vec{\kappa})(\vec{A} \cdot \vec{\kappa})$. The obvious $U$-spin vector is related to the $d$-s mass matrix,

$$
\mathcal{M}=\left(\begin{array}{cc}
m_{s} & 0 \\
0 & m_{d}
\end{array}\right) .
$$

However, here is the point. At short distances, the mass matrix, (2.8), transforms as a $(2,2)$ under (2.2). Thus the $U$-spin vector must be constructed from the $S U(2)_{D R}$ singlet combination, $\mathcal{M M}^{\dagger}$, and the $U$-spin violation is proportional to

$$
\operatorname{tr}\left(\tau_{3} \mathcal{M} \mathcal{M}^{\dagger}\right)=m_{s}^{2}-m_{d}^{2} \approx m_{s}^{2}
$$

However, at long distances, the chiral $S U(2)_{D L} \times S U(2)_{D R}$ symmetry is spontaneously broken by the strong QCD interactions down to the vector diagonal $U$-spin. At long distances, a $U$-spin triplet can be constructed from $\mathcal{M} \Sigma^{\dagger}$, where $\Sigma$ is the chiral symmetry breaking condensate. Thus we might expect long distance contributions proportional to $m_{s}^{3}$ and $m_{s}^{2}$, rather than the $m_{s}^{4}$ that is required for the short distance contributions.

\section{The Heavy Quark Effective Theory}

The description of $D-\bar{D}$ mixing in the language of the HQEFT that we will develop in this section may seem peculiar at first. It is well to remember that the HQEFT is nothing really profound. All the results could be equally well derived in the conventional QCD theory, so long as we take careful account of where large momenta are flowing. The HQEFT is simply a convenience. It allows us to use the framework of effective field theories to do automatically the work of isolating large momenta in the full theory. Of course I should admit again that in all of this we are assuming that $m_{c}$ is large, in particular that there is a gap between $m_{c}$ and the scale, $\approx 1 \mathrm{GeV}$ of chiral symmetry breaking. In reality, there is hardly any gap at all. Nature has played an exquisite joke on the practitioners of the HQEFT, because it is really probably only for the $b$ quark that the techniques of HQEFT apply without very large corrections. Nevertheless, the HQEFT may be useful even though the corrections are often large, just as $S U(3)$ and chiral $S U(3) \times S U(3)$ are useful, even though the $m_{s}$ is not very small.

When you go below the $c$ mass scale and go to the heavy quark effective theory, the charm changing nonleptonic operators disappear from the Lagrangian of the effective theory, because any 
such term gives rise to a light colored particle carrying momentum of the order of $m_{c}$. Thus below the scale $m_{c}$, there are no charm changing nonleptonic interactions in the effective theory. Now that seems a little strange, because the effects of the nonleptonic decays must still be there. Presumably the way it works is that when you match at two loops, the heavy quark propagator will acquire a phase because of the width,

$$
\frac{1}{v k-\Delta m-i \Gamma}
$$

where $\Gamma$ is the width and $\Delta m$ is the difference between the quark mass and the mass used to define the velocity dependent fields. Thus in this order, the heavy quark kinetic energy term is complex. It should not be surprising that the kinetic energy term for the heavy quark in the HQEFT is non-unitary. Probability is conserved in the HQEFT only if the heavy quark is absolutely stable. The width, $\Gamma$, describes the leakage of the heavy quarks out of the HQEFT.

At any rate, the nonleptonic interactions are not there to produce $D-\bar{D}$ mixing in the heavy quark theory. The moral is that there are no "dispersive" contributions to the mixing from physics below the scale $m_{c}$. In the heavy quark effective theory, all the mixing comes from the matrix element of the $\Delta c=2$ operators produced by matching at the scale $m_{c}$. In the language of the full theory, there is always a momentum of order $m_{c}$ flowing through the graphs that produce $D \bar{D}$ mixing. Of course, there are still two types of contributions:

1. Contributions from matching of 4-quark operators produced at scales above $m_{c}$ (for example, from eliminating the $b$ quark if we do not ignore $s_{2}$ and $\left.s_{3}\right)$;

2. Contributions from two insertions of the nonleptonic $\Delta c=1$ part of the Hamiltonian at the scale $m_{c}$.

The first are certainly short-distance contributions. The second must include the long-distance contributions, but in the heavy quark effective theory in leading order in $1 / m_{c}$, these effects just contribute to the same kinds of $\Delta c=2$ operators. As we will see below, the interesting long-distance contributions are actually non-leading in $1 / m_{c}$.

The type 2 contribution to leading order in $1 / m_{c}$ is a one loop matching correction calculated by doing the box diagram calculation with momentum $m_{c} v$ in the external $c$ lines, and therefore flowing through the diagram, as shown in figure 1. It is easy to see that this is proportional to (in lowest order in $1 / m_{c}$ and ignoring $m_{d}$ )

$$
\frac{1}{16 \pi^{2}} \sin ^{2} \theta \cos ^{2} \theta G_{F}^{2} \frac{m_{s}^{4}}{m_{c}^{2}}
$$


so it is small. There is no enhancement. You cannot even use HQEFT to relate this contribution to $B \bar{B}$ mixing - there are two operators, because of the $v^{\mu}$ dependence. That is, in addition to the usual operator,

$$
\left(\overline{c_{v}} \gamma^{\mu} u_{L}\right)\left(\overline{\bar{c}_{v}} \gamma_{\mu} u_{L}\right)
$$

(where we are using the notation of [6] in which $\underline{c}_{v}$ represents the heavy antiquark field, there is also a contribution proportional to the operator

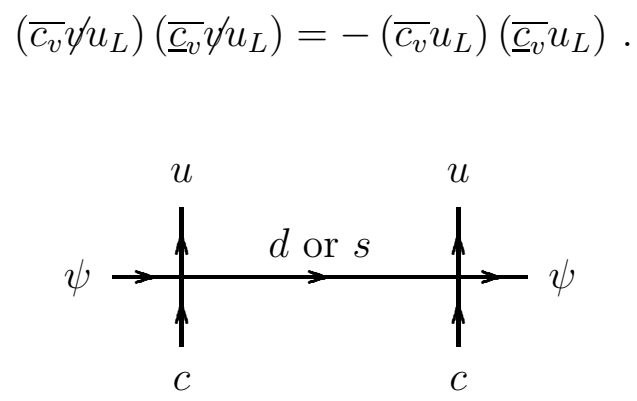

Figure 4: Feynman graph contributing to tree level matching to leading order in $\alpha_{s}$.

More interesting are the operators produced by the tree level matching. These are nonleading in $1 / m_{c}$, but as we will see, they probably give the dominant contribution to the mixing. First consider mixing to a 6 -quark operator from the diagram shown in figure 4 . This gives contributions such as

$$
\begin{aligned}
& \sin \theta \cos \theta \frac{G_{F}^{2}}{2} \frac{m_{s}^{2}}{m_{c}^{3}}\left(\underline{\bar{c}}_{v} \gamma^{\mu} \gamma \gamma^{\nu} u_{L}\right)\left(\bar{\psi} \gamma_{\mu} u_{L}\right) \vec{\kappa} \cdot \vec{\tau}\left(\overline{c_{v}} \gamma_{\nu} \psi_{L}\right) \\
& \left.\left.\psi \rightarrow\right|_{c} ^{u} \psi \psi \psi \rightarrow\right|_{c} ^{u}
\end{aligned}
$$

Figure 5: Feynman graph contributing to tree level matching to first order in $\alpha_{s}$.

In addition, there are 8-quark operators produced by graphs like that shown in figure 5. These contributions have no explicit factors of $m_{s}$. For example, the contribution of the graph of figure 5 looks like

$$
\frac{g_{s}^{2} G_{F}^{2}}{2 m_{c}^{4}}\left(\bar{\psi} \gamma^{\mu} u_{L}\right) \vec{\kappa} \cdot \vec{\tau}\left(\underline{\bar{c}}_{v} \gamma_{\alpha} \psi \gamma_{\mu} T_{a} u_{L}\right)\left(\bar{\psi} \gamma_{\nu} u_{L}\right) \vec{\kappa} \cdot \vec{\tau}\left(\overline{c_{v}} \gamma^{\alpha} \psi \gamma^{\nu} T_{a} \psi_{L}\right)
$$


Because of the gluon exchange, we will see that contributions of this type are likely to be less important than the contribution of (3.14).

As discussed in the previous section, the reason that these contributions can be important in spite of the extra factors of $1 / m_{c}$ is that the matrix element of the $U$-spin vector operator $\bar{\psi}_{L} \vec{\kappa} \cdot \vec{\tau} \psi_{L}$ in a low energy hadronic state is proportional to only one power of $m_{s}$. This is possible because the chiral $U$-spin symmetry, (2.2), is spontaneously broken. Thus the GIM suppression from these long-distance contributions is proportional to $m_{s} \Lambda$ (where $\Lambda \approx 1 \mathrm{GeV}$ is the chiral symmetry breaking scale), while the GIM suppression in the leading operators (including all the short-distance contributions) is guaranteed to be proportional to $m_{s}^{2}$, because the $S U(3)_{D R}$ is unbroken except by the mass term. Thus while the "long-distance" contributions have more factors of $1 / m_{c}$, they have fewer factors of $m_{s}$.

In particular, let us use naive dimensional analysis [7] (NDA) to estimate the matrix elements of the different operators. Dimensional analysis gives the usual estimate of (3.12) and (3.13) with the NDA relation

$$
f_{D} \approx f\left(\frac{\Lambda}{m_{c}}\right)^{1 / 2} .
$$

The interesting question is the ratio of the contribution of (3.14) to these. The difference is that coefficient of (3.14) has an extra factor of

$$
\frac{16 \pi^{2}}{m_{s}^{2} m_{c}}
$$

and the extra U-spin vector operator,

$$
d \bar{d}-s \bar{s} .
$$

NDA suggests that (3.18) has a matrix element of order

$$
m_{s} f^{2}
$$

so that the ratio of the contribution of (3.14) to (3.12) is expected to be of order

$$
\frac{16 \pi^{2}}{m_{s}^{2} m_{c}} m_{s} f^{2}=\frac{4 \pi f}{m_{s}} \frac{4 \pi f}{m_{c}} .
$$

On the other hand, the ratio of the contribution of (3.15) to (3.12) is expected to be proportional to

$$
\frac{\alpha_{s}}{4 \pi}\left(\frac{4 \pi f}{m_{s}} \frac{4 \pi f}{m_{c}}\right)^{2}
$$




\section{Conclusions}

The relation, (3.20), shows a modest enhancement of the "long-distance" contributions to $D-\bar{D}$ mixing compared to the short-distance contributions. Of course, the effect could always be magnified by an unexpectedly large matrix element of the six quark operator. Certainly, it is reasonable to expect some additional enhancement, given the large $S U(3)$ violation in $D^{0}$ decays. Nevertheless, (3.20) and (3.21) suggest something quite interesting about the calculation of [2, 3]. If you look at the contribution of any particular set of states (related by their $S U(3)$ properties) to the mixing (for example, all pairs of two light pseudoscalars), ala [2, 3], you would expect a contribution of order

$m_{s}^{2}$, because the final states feel the long distance QCD interactions in which the chiral symmetry is spontaneously broken. But the HQEFT analysis of the previous section shows that the full order $m_{s}^{2}$ contribution to the mixing is actually suppressed by an additional factor of $\alpha_{s} / 4 \pi$. Thus it is reasonable to expect cancellations between the contributions of different types of intermediate states. The dominant remaining contribution, proportional to $m_{s}^{3}$, is in some sense intermediate between the short distance contributions and the "dispersive" contributions of [2, 3].

\section{Acknowledgements}

I am grateful to Chris Carone, Rowan Hamilton, Thorsten Ohl, Gulia Ricciardi and Elizabeth Simmons for useful discussions. Research supported in part by the National Science Foundation under Grant \#PHY-8714654 and by the Texas National Research Laboratory Commission, under Grant \#RGFY9206.

\section{References}

[1] N. Isgur and M. B. Wise, Phys. Lett. 232B (1989) 113; 208B (1988) 504; E. Eichten and B. Hill, Phys. Lett. 234B (1990) 511; B. Grinstein, Nucl. Phys. B339 (1990) 253; H. Georgi, Phys. Lett. B240 (1990) 447.

[2] J.F. Donoghue et al., Phys. Rev. D33 (1986) 179.

[3] L. Wolfenstein, Phys. Lett. 164B (1985) 170.

[4] F. Gilman and M.B. Wise, Phys. Rev. D20 (1979) 2392. 
[5] S. L. Glashow, J. Iliopoulos and L. Maiani, Phys. Rev. D2 (1970) 1285.

[6] H. Georgi, Heavy Quark Effective Field Theory, in Proc. of the Theoretical Advanced Study Institute 1991, ed. R.K. Ellis, C.T. Hill and J.D. Lykken (World Scientific, Singapore, 1992) p. 589.

[7] A. Manohar and H. Georgi, Nucl. Phys. B234 (1984) 189, and H. Georgi and L. Randall, Nucl. Phys. B276 (1986) 241. 